\title{
Color tunable organic light-emitting diodes by using europium organometallic complex
}

\author{
C. J. Liang and Wallace C. H. Choy ${ }^{\text {a) }}$ \\ Department of Electrical and Electronic Engineering, The University of Hong Kong, Pokfulam Road, Hong \\ Kong
}

(Received 12 September 2006; accepted 7 November 2006; published online 19 December 2006)

\begin{abstract}
Using the rare-earth special feature of a sharp emission spectrum, voltage-controlled continuous color tuning of organic light-emitting diodes is achieved. Europium(dibenzoylmethanato) ${ }_{3}$ (bathophenanthroline) is used as the strategic starting point close to the red corner of the Commission International de I'Eclairage chromaticity diagram for a wide color tuning. The end point and path of the color tuning can be engineered by doping the hole-transport emitting layer with dyes. The mechanisms of color tuning have been investigated and explained by the efficiency reduction of the europium complex and the extension of carrier recombination zone with driving voltage. The effect of exciplex on the color tuning is also studied. (C) 2006 American Institute of Physics. [DOI: 10.1063/1.2405420]
\end{abstract}

Organic light-emitting diode (OLED) is one of the promising technologies for light display applications. In order to achieve a full-color pixel in display applications, complicated techniques have to be used to accurately fabricate three OLED structures for red, green, and blue emissions in small areas. ${ }^{1,2}$ It will be highly desirable if a simply single OLED can emit light with a wide range of colors, continuously tunable by changing the voltage for simplifying the fabrication techniques of multi- and full-color devices. Voltagecontrolled color tuning has been reported in OLEDs using small molecules $^{3-5}$ and polymers, ${ }^{6-8}$ as well as in lightemitting electrochemical cells. ${ }^{9,10}$ However, more efforts are needed to expand the tuning range and to understand the underlying mechanism.

Rare-earth organometallic complexes have the interesting features of sharp emission spectrum and theoretically high electroluminescent (EL) efficiency. Indeed, high quantum efficiency $(\eta)$ of EL of $7.5 \%$ was obtained in europium (Eu) complex ${ }^{11}$ at low current density. In this letter, a Eu complex is used as the strategic starting point for the color tuning. The sharp red emission close to the corner of the Commission International de I'Eclairage (CIE) chromaticity diagram offers a large space for color tuning. The OLEDs containing $\mathrm{Eu}$ (dibenzoylmethanato) ${ }_{3}$ (bathophenanthroline) $\left[\mathrm{Eu}(\mathrm{DBM})_{3}\right.$ bath] acting as the emitting and electrontransport (ET) layers, and $N, N^{\prime}$-bis(2-naphtalenyl)$N$ - $N^{\prime}$-bis(phenylbenzidine) (NPB) as the hole-transport layer are studied. Transient EL, current efficiency, and $\mathrm{Eu}^{3+}$ emission $\eta$ are investigated to clarify the mechanism of the color tuning. The effects of exciplex on the color tuning are also addressed. Depending on the emission color of dyes, doping the NPB layer offers various routes of the color tuning on the CIE diagram.

The basic device structure (device A) is indium tin oxide $(\mathrm{ITO}) / \mathrm{NPB}(40 \mathrm{~nm}) / \mathrm{Eu}(\mathrm{DBM})_{3} \operatorname{bath}(40 \mathrm{~nm}) / \mathrm{Alq}_{3}$ $(20 \mathrm{~nm}) / \mathrm{LiF} / \mathrm{Al}$. $\mathrm{Eu}(\mathrm{DBM})_{3}$ bath has been shown to have good ET and emitting properties in the earlier studies. ${ }^{12}$ The multilayer of $\mathrm{Alq}_{3} / \mathrm{LiF} / \mathrm{Al}$ acts as a composite electron-

\footnotetext{
a) Author to whom correspondence should be addressed: FAX: (852) 2559-
} 8738; electronic mail: chchoy@eee.hku.hk injection electrode. It should be noted that the ET properties and thus the brightness and $\eta$ of OLEDs can be further enhanced by cohosting the $\mathrm{Alq}_{3}$ based ET layer ${ }^{13}$ although the study is out of the scope of this letter. All the organics and the $\mathrm{LiF} / \mathrm{Al}$ cathode are deposited by thermal vacuum deposition at a pressure of $10^{-6}$ Torr. Transient EL was studied by applying a voltage pulse across the device. By using a monochromator for wavelength selection, the transient spectrum was detected by a photomultiplier and an oscilloscope.

The EL spectra of device A are shown in Fig. 1. At the voltage of $6 \mathrm{~V}$, the EL spectrum shows the solely sharp emission from the Eu complex. At higher voltages a board emission in the shorter-wavelength region appears and its intensity continuously strengthens. Comparing with the photoluminescent (PL) spectrum of NPB, the board emission consists of two parts: one is the emission at $\sim 450 \mathrm{~nm}$ from $\mathrm{NPB}$; the other at $\sim 500 \mathrm{~nm}$ can be due to either $\mathrm{Alq}_{3}$ or the exciplex forming at the interface between two organic layers. To study the origin of the emission, we replace $\mathrm{Alq}_{3}$ by UV emissive 2, $2^{\prime}, 2^{\prime \prime}$-(1,3,5-benzinetriyl)-tris (1-phenyl-1-Hbenzimidazole). The results show that the emission at $\sim 500 \mathrm{~nm}$ still exists. This implies that it is the exciplex



FIG. 1. EL spectra of device A. They are normalized to the Eu peak at $615 \mathrm{~nm}$. The solid line is the PL spectrum of NPB film. 


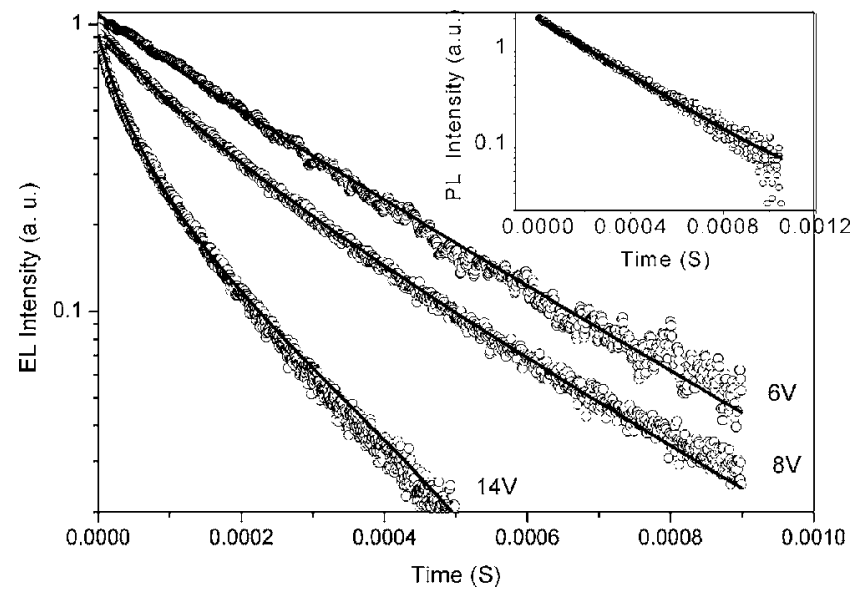

FIG. 2. Transient EL of $\mathrm{Eu}^{3+}$ ions in device A; experimental data (open circles) and fitting curves (solid lines) are obtained by using Eq. (1) with $K=0.7 \times 10^{3}, 3.5 \times 10^{3}$, and $1.8 \times 10^{4} \mathrm{~s}^{-1}$ for 6,8 , and $14 \mathrm{~V}$, respectively. The fitting curves are normalized at $t=0$. The inset shows the decay of $\mathrm{Eu}(\mathrm{DBM})_{3}$ bath characterized by $\mathrm{N}_{2}$ pulse laser excitation.

emission from the interface of NPB and the Eu complex. The NPB emission becomes dominantly stronger than the exciplex emission as the voltage is further increased. As a result, continuously reversible color tuning from pure red to blue is obtained.

Two reasons may cause the distinct EL spectrum changes. One is the $\eta$ decrease of $E u(\mathrm{DBM})_{3}$ bath at high voltages; the other is the shift of recombination zone in the device. Transient EL has been used to understand the biexcitonic quench and $\eta$ decrease of the $\mathrm{Eu}^{3+}$ emission in the process of color tuning. Considering that interaction between two excited $\mathrm{Eu}^{3+}$ ions causes a quenching channel in the decay process of the excited $\mathrm{Eu}^{3+}$ ions, the transient intensity $L(t)$ violates the typical exponential decay rule and follows: ${ }^{14}$

$$
L(t)=\frac{L(0)}{(1+K \tau) e^{t / \tau}-K \tau},
$$

where $\tau$ is the lifetime of the excited ions due to emissive decay, $L(0)$ is the initial emission intensity, and $K$ is the biexcitonic quench rate. Thus $\eta$ of the $\mathrm{Eu}^{3+}$ ions is

$$
\eta=\frac{1}{1+K \tau}
$$

where $\tau$ can be obtained from the transient PL and EL under weak excitation intensity. $K$ can be determined by fitting the transient EL decay data with Eq (1).

The decay of $\mathrm{Eu}^{3+}$ ions after excitation of pulse voltages are shown in Fig. 2. We set the measured wavelength at $614 \mathrm{~nm}$ such that the detected signals only describe the decay of $\mathrm{Eu}^{3+}$ ions. The inset of Fig. 2 shows the PL decay of $\mathrm{Eu}(\mathrm{DBM})_{3}$ bath. The exponential decay fitting gives $\tau$ $\sim 300 \mu$ s. Using Eq. (1), the decay process is fitted as shown in Fig. 2. The values of $K$ are $0.7 \times 10^{3}, 3.5 \times 10^{3}$, and 1.8 $\times 10^{4} \mathrm{~s}^{-1}$ for 6,8 , and $14 \mathrm{~V}$, respectively. According to Eq. (2), $\eta$ of $\mathrm{Eu}^{3+}$ emission are $0.82,0.49$, and 0.16 for 6,8 , and $14 \mathrm{~V}$, respectively. These clarify that the biexcitonic quench does happen and makes $\eta$ of $\mathrm{Eu}^{3+}$ emission decrease significantly at higher voltages and therefore is useful for achieving the color tuning.

Figure 3 compares the evolution trends of $\eta$ of the exDownloaded 20 Apr 2007 to 147.8.143.135. Redistribution subject



FIG. 3. Current efficiency (filled circle) of device A and $\eta$ of the excited $\mathrm{Eu}^{3+}$ ions (open triangle). The $\mathrm{Eu}^{3+} \eta$ is factorized to the same value as the device current efficiency at $6 \mathrm{~V}$. The inset is the energy diagram of device A.

cited $\mathrm{Eu}^{3+}$ ions and the measured current efficiency of device A. The results show that the two trends of reduction with increasing voltage are similar. However, the reduction of the device efficiency is more drastic than that of the $\mathrm{Eu}^{3+} \eta$, implying that the recombination zone changes. The inset of Fig. 3 shows the energy diagram of device A. At the interface of NPB and $\mathrm{Eu}(\mathrm{DBM})_{3}$ bath the energy barriers for electrons to enter NPB layer and for holes to enter $\mathrm{Eu}(\mathrm{DBM})_{3}$ bath are almost the same. Therefore the shift or extension of recom-

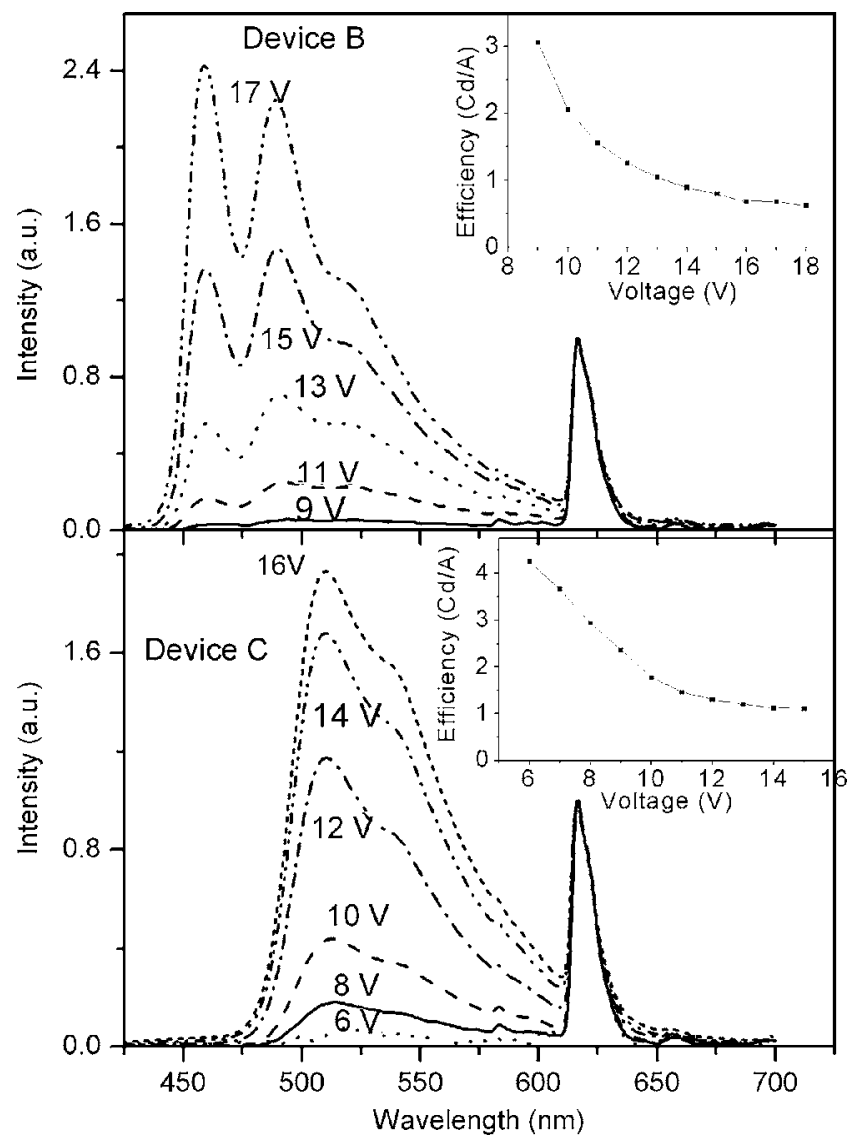

FIG. 4. EL spectra of devices B and C. They are normalized to the Eu peak

at $615 \mathrm{~nm}$. The insets show the current efficiency of the devices.
AIP license or copyright, see http://apl.aip.org/apl/copyright.jsp 


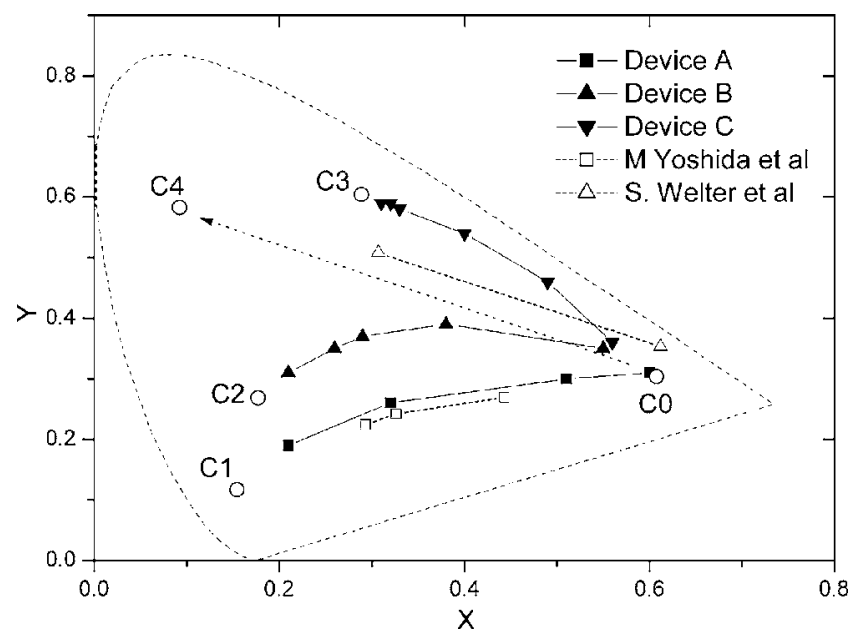

FIG. 5. Color tuning of devices A, B, and $\mathrm{C}$ on the CIE chromaticity diagram. The circles $\mathrm{C} 0, \mathrm{C} 1, \mathrm{C} 2$, and $\mathrm{C} 3$ are the emission colors of pristine $\mathrm{Eu}(\mathrm{DBM})_{3}$ bath, NPB, perylene doped NPB, and C545T doped NPB, respectively. C4 is the emission color of a supposed fluorescent dye doped NPB. The results denoted by $\square$ and $\Delta$ are extracted from Refs. 3 and 10, respectively.

bination zone at the interface with the driving voltages is greatly possible. As shown in Fig. 1, since the emission of $\mathrm{Eu}(\mathrm{DBM})_{3}$ bath is still present at high driving voltage, it can be readily considered that the extension of recombination zone occurs in the device, i.e., the carrier recombination zone mainly locates in the $\mathrm{Eu}(\mathrm{DBM})_{3}$ bath layer at low bias and it extends across the interface into the NPB layer at higher voltages. This is the second major reason for the color tuning.

Since NPB can serve as the host for many fluorescent dopants, it is possible to change the tuning color by using dopants. Two typical dopants of perylene and coumarin 545T (C545T) for blue and green emissions respectively, are used to change the tuning color. The structures of $\mathrm{ITO} / \mathrm{NPB}(20 \mathrm{~nm}) / \mathrm{NPB}: \operatorname{dopant}(20 \mathrm{~nm}) / \mathrm{Eu}(\mathrm{DBM})_{3}$ bath $(40 \mathrm{~nm}) /(20 \mathrm{~nm}) / \mathrm{LiF} / \mathrm{Al}$ with perylene and C545T as dopants for devices B and C, respectively, have been fabricated and their EL spectra are shown in Fig. 4. The results show that the emission of NPB is replaced by the dopants, and the color tuning routes are changed accordingly. In addition, the insets of Fig. 4 show that the reduction of current efficiencies of devices B and C is slower than that of devices A, and the efficiencies at higher voltages are improved due to the better emitting properties of the doped NPB layers. The color tuning of devices A, B, and $\mathrm{C}$ are summarized in Fig. 5. The CIE coordinates of the pristine materials are also plotted as a reference. $\mathrm{C} 0$ and $\mathrm{C} 1$ are the $\mathrm{CIE}$ coordinates of $\mathrm{Eu}^{3+}$ and NPB emissions, respectively. Device A shows the color tuning along the line starting from $\mathrm{C} 0$ and moving towards $\mathrm{C} 1$. Doping NPB with perylene (device B) and C545T (device C) changes the destination to $\mathrm{C} 2$ (perylene emission) and $\mathrm{C} 3$ (C545T emission), respectively. Also shown in Fig. 5 are the typical color tunable results of others. ${ }^{3,10}$ The color tunabilities of devices $\mathrm{A}, \mathrm{B}$, and $\mathrm{C}$ are considerable as compared to them. Consequently, the ending-color adjustable and widerange color tunable scheme is achieved in the simple device configuration. This confirms that we can engineer the color tuning route of the device. For instance, if a dopant in NPB emits a color at $\mathrm{C} 4$, we could obtain the color tuning route starting from $\mathrm{C} 0$ and ending towards $\mathrm{C} 4$. It is worth to note that when the color tuning is merely caused by intensity changes from two emitting species, the tuning route should be a straight line linked between the two destination colors. In the result, the tuning route is curved. This can be explained by the emission from a third party which is the exciplex emission as discussed previously.

In conclusion, making use of $\mathrm{Eu}^{3+}$ featuring a sharp red emission, reversible voltage-controlled continuous color tuning is achieved in the OLEDs by using $\mathrm{Eu}(\mathrm{DBM})_{3}$ bath as the strategic starting point close to the red corner of the CIE chromaticity diagram. The ending-color adjustable and widerange color tuning scheme is achieved by doping the NPB layer with dyes. The exciplex emission makes the color tuning path curved instead of a straight line. In our experiments, the $\eta$ reduction of the Eu complex and extension of carrier recombination zone at higher bias have been shown to be the major reasons for achieving the color tuning. The stability of Eu complex in OLED is still a challenge, further work of new chemically stable Eu complexes are needed in the field.

The auther acknowledge the support from UDF grant and the strategic research grant in organic optoelectronics of HKU and the grant (No. 14300.324.01) from the Research Grant Council of the HK Special Administrative Region, China. We thank the help of P.T. Lai and Y. Luo for setting up the laboratory and W.L. Li for synthesizing $\mathrm{Eu}(\mathrm{DBM})_{3}$ bath. The authors thank e-Ray Optoelectronics for supplying part of the organic materials at special prices.

${ }^{1}$ H. Kobayashi, S. Kanbe, S. Seki, H. Kigchi, M. Kimura, I. Yudasaka, S. Miyashita, T. Shimoda, C. R. Towns, J. H. Burroughes, and R. H. Friend, Synth. Met. 111-112, 125 (2000).

${ }^{2}$ J. Birnstock, J. Blassing, A. Hunze, M. Scheffel, M. Stoßel, K. Heuser, G. Wittmann, J. Worle, and A. Winnacker, Appl. Phys. Lett. 78, 3905 (2001).

${ }^{3}$ M. Yoshida, A. Fujii, Y. Ohmori, and K. Yoshino, Appl. Phys. Lett. 69, 734 (1996).

${ }^{4}$ J. Kalinowski, P. Di Marco, M. Cocchi, N. Camaioni, and J. Duff, Appl. Phys. Lett. 68, 2317 (1996).

${ }^{5}$ R. Reyes, M. Cremona, E. E. S. Teotonio, H. F. Brito, and O. L. Malta, Chem. Phys. Lett. 396, 54 (2004).

${ }^{6}$ M. Granstrom and O. Inganas, Appl. Phys. Lett. 68, 147 (1996).

${ }^{7}$ Y. Z. Wang, R. G. Sun, F. Meghdadi, G. Leising, and A. J. Epstein, Appl. Phys. Lett. 74, 3613 (1999)

${ }^{8}$ C. C. Huang, H. F. Meng, G. K. Ho, C. H. Chen, C. S. Hsu, J. H. Huang, S. F. Horng, B. X. Chen, and L. C. Chen, Appl. Phys. Lett. 84, 1195 (2004).

${ }^{9}$ Y. Yang and Q. Pei, Appl. Phys. Lett. 68, 2708 (1996).

${ }^{10} \mathrm{~S}$. Welter, K. Brunner, J. W. Hofstraat, and L. De Cola, Nature (London) 421, 54 (2003).

${ }^{11}$ T. W. Canzler and J. Kido, Org. Electron. 7, 29 (2006).

${ }^{12}$ C. J. Liang, D. Zhao, Z. R. Hong, D. X. Zhao, X. Y. Liu, W. L. Li, J. B. Peng, J. Q. Yu, C. S. Lee, and S. T. Lee, Appl. Phys. Lett. 76, 67 (2000); Z. R. Hong, C. J. Liang, R. G. Li, W. L. Li, D. Zhao, D. Fan, D. Y. Wang, B. Chu, F. X. Zang, L. S. Hong, and S. T. Lee, Adv. Mater. (Weinheim, Ger.) 13, 1241 (2001).

${ }^{13}$ H. H. Fong, W. C. H. Choy, K. N. Hui, and Y. J. Liang, Appl. Phys. Lett. 88, 113510 (2006).

${ }^{14}$ C. J. Liang, T. C. Wong, L. S. Hung, S. T. Lee, Z. R. Hong, and W. L. Li, J. Phys. D 34, L61 (2001). 